\title{
Association of Intestinal Dysbiosis Problem with Initiation and Development of Somatic Diseases
}

\author{
Kulchitsky Vladimir ${ }^{1 *}$, Zamaro Alexandra ${ }^{1}$, Huo Po $^{2}$, Li Junbo ${ }^{2}$, Chen Xinrui ${ }^{2}$ and Koulchitsky Stanislav ${ }^{3}$ \\ ${ }^{1}$ Institute of Physiology, National Academy of Sciences, Minsk, Belarus \\ ${ }^{2}$ Zhejiang University of Science \& Technology, Hangzhou, China \\ ${ }^{3}$ Liege University, Liege, Belgium
}

Received: 制 August 21, 2018; Published: 眥 August 23, 2018

*Corresponding author: Vladimir Kulchitsky, Scientific Director, Institute of Physiology, National Academy of Sciences of Belarus, 28 Akademicheskaya Street, Minsk, 220072, Belarus

Keywords: Microbiota; Gastrointestinal Tract; Endotoxemia; Illness

\section{Introduction}

Deficiency of solutions of key issues of initiation, development, prophylaxis and therapy of many somatic diseases results in the search for new external and internal causes of pathological processes manifestation in various functional systems of the organism. Recently researchers paid attention to internal - not external - cause of obesity, diabetes, atherosclerosis, cardiovascular syndrome, allergy, asthma, and neurodegenerative diseases development [1-4]. It was suggested that this trigger mechanism is localized in microbiota of human gastrointestinal tract [1]. This opinion is based on facts of pronounced dysbiosis and intestinal dysbacteriosis revealed in patients with these somatic diseases [14]. What is the initial cause - development of pathological processes in the intestine accompanied with initiation of somatic pathology or, alternatively, developing somatic pathology is accompanied with intestinal dysbiosis?

\section{Intestinal Dysbiosis and Somatic Pathological Processes}

Researchers' opinions divided, however more and more blame intestinal dysbiosis for initiation of somatic pathological processes [1,5]. The amount of microorganisms in the intestine is compatible with the amount of cells in human body and reaches 37 trillion units [1]. Therefore, the common genetic kit of microbiota and its unpredictable adaptive and sometimes aggressive abilities look quite impressive. Naturally, microbiota and human organism stay in harmony. Dysbiosis and dysbacteriosis are accompanied with violation of relationships between the host and microorganisms. Imbalance between aerobes and anaerobes in the intestine and change of amount and composition of certain pools of microorganisms - all these lead to appearance of conditions for translocation of natural intestinal endotoxins and metabolism products from intestinal lumen to bloodstream $[1,5,6]$. The emphasis is placed on negative consequences of dysbiosis development for functioning of endocrine organs, brain, kidneys, liver, heart and vessels, skeletal muscles $[1,3,4]$.

Decarboxylation and deamination processes are disturbed in dysbiosis. These processes are naturally aimed at lysis of amino acids [1]. Excess of amino acids in the intestine serves as nutrients for rectal microflora. Bacterial enzymes break down excess of amino acids converting them into amines, phenols, indole, skatole, hydrogen sulphide and other poisonous for human organism substances. Phenol and cresol are formed from amino acid tyrosine, indole and skatole - from tryptophan. Scientists noticed that "drop" of certain amount of blood, for example in stress, from hepatic-portal system to systemic circulation is also accompanied with translocation of endotoxin, indole, skatole, phenol and cresol. Endotoxin (for example, E.coli lipopolysaccharide) binds with TLR4 and naturally activates immunity of host's organism through cascade of processes involving CD14 and proinflammatory cytokines (IL-1 $\beta$, IL6, TNF) [1,7]. However, excessive endotoxin translocation into blood leads to fever development by proinflammatory cytokines together with local inflammatory process in "vulnerable" regions. Such local inflammatory processes are accompanied with formation of atherosclerotic plaques, disturbance of endocrine organs functioning and even malignant transformation of cells $[1,8,9]$. Integrity of negative events in the organism during dysbiosis development allowed us proposing the term - "syndrome of intestinal dysbiosis". The search for "intestinal and dysbiosis' and syndrome" in PubMed revealed only two articles on August 21, 2018 [10,11]. Endotoxemia expectedly enhances during initial development of such syndrome in the intestine. Uncontrolled endotoxemia results in the development of local and systemic inflammatory and metabolic pathological processes in many organs and systems. In this case proposed term "syndrome of intestinal dysbiosis" differs from the term "metabolic syndrome" 
by distinct criterion, namely severity of endotoxemia. Systematic monitoring of endotoxemia grade is the basis for prophylaxis and correction of developing pathological processes. By the way, first of all pathological processes begin appearing in those organs and systems which have specific functional and structural weakness to endotoxemia resistance.

More and more endotoxin and other metabolites portions fall into bloodstream in violation of liver functions. Such processes are eventuallyaccompanied with developmentofpersistentpathological processes leading to manifestation of diabetes, atherosclerosis, kidney failure, and destruction of brain neurons. The facts of low content of endotoxin in blood of long-livers compared to working middle-aged volunteers [12] is important additional argument in favor of delivered hypothesis on endotoxemia role in manifestation of pathological processes contributing to development of diabetes, atherosclerosis and other somatic diseases $[1,3,4,8,9]$.

There are facts of endotoxin translocation from intestine into bloodstream during simulation of disturbed autonomous regulation of gastrointestinal tract $[13,14]$. One of the models includes selective cuts of intestinal branches of vagus nerve with subsequent examination of systemic and local influences of such cut on fever development $[13,15]$. The role of vagus nerve system in endotoxemia grade reporting to various brain regions was established in these experiments [13-15]. The role of neurohumoral and endocrine regulation in relationships between microbiota and host's organism should be detailed in future.

\section{Conclusion}

In fact, it is proposed to bring in key element in the vision of pathological mechanisms of somatic diseases development functional state of intestinal microbiota. The grade of intestinal dysbiosis affects formation of conditions for endotoxemia initiation and manifestation of pathological processes accompanied with development of systemic and local sites of inflammation in blood vessel walls and various organs. Confirmation of this hypothesis will allow creating reasonable methods of pathological processes correction at the stage of human intestine. Positive effects of different diets, e.g. Mediterranean one, significance of Chinese traditions of tea-drinking, relevance of products' quality in food retail chains, parenting of nutrition ritual from an early age, etc. should be reviewed once again.

\section{Acknowledgement}

This analysis was funded by grant of 000 "Synergy", and by grant of BRFFI A18AC-025 (2018-2019).

\section{Conflict of Interest}

All listed authors concur with the submission of the manuscript; all authors have approved the final version. The authors have no financial or personal conflicts of interest.

\section{References}

1. Spielman LJ, Gibson DL, Klegeris A (2018) Unhealthy gut, unhealthy brain: The role of the intestinal microbiota in neurodegenerative diseases. Neurochem Int S0197-0186(18): 30198-30190.

2. Gagliardi A, Totino V, Cacciotti F, Iebba V, Neroni B, et al. (2018) Rebuilding the Gut Microbiota Ecosystem. Int J Environ Res Public Health 15(8): E1679.

3. Iebba V, Nicoletti M, Schippa S (2012) Gut microbiota and the immune system: an intimate partnership in health and disease. Int J Immunopathol Pharmacol 25(4): 823-833.

4. Vespasiani-Gentilucci U, Gallo P, Picardi A (2018) The role of intestinal microbiota in the pathogenesis of NAFLD: starting points for intervention. Arch Med Sci 14(3): 701-706.

5. Romanovsky AA, Kulchitsky VA, Akulich NV, Koulchitsky SV, Simons CT, et al. (1996) First and second phases of biphasic fever: two sequential stages of the sickness syndrome? Am J Physiol 271: R244-R253.

6. Romanovsky AA, Sugimoto N, Szekely M, Kulchitsky VA (1997) Febrile responsiveness of vagotomized rats is suppressed even in the absent of malnutrition. Am J Physiol 273: R777-R783.

7. Wanner SP, Yoshida K, Kulchitsky VA, Ivanov AI, Kanosue K, et al. (2013) Lipopolysaccharide induced neuronal activation in the paraventricular and dorsomedial hypothalamus depends on ambient temperature. PLoS One 8(9): e75733.

8. González-Sarrías A, Romo-Vaquero M, García-Villalba R, Cortés-Martín A, Selma MV, et al. (2018) The Endotoxemia Marker LipopolysaccharideBinding Protein is Reduced in Overweight-Obese Subjects Consuming Pomegranate Extract by Modulating the Gut Microbiota: A Randomized Clinical Trial. Mol Nutr Food Res 62(11): e1800160.

9. González-Sarrías A, Núñez-Sánchez MA, Ávila-Gálvez MA, Monedero-Saiz T, Rodríguez-Gil FJ, et al. (2018) Consumption of pomegranate decreases plasma lipopolysaccharide-binding protein levels, a marker of metabolic endotoxemia, in patients with newly diagnosed colorectal cancer: A randomized controlled clinical trial. Food Funct 9(5): 2617-2622.

10. Roberfroid M, Gibson GR, Hoyles L, McCartney AL, Rastall R, et.al. (2010) Prebiotic effects: metabolic and health benefits. Br J Nutr 104(2): S1S63.

11. Weese JS, Nichols J, Jalali M, Litster A (2015) The rectal microbiota of cats infected with feline immunodeficiency virus infection and uninfected controls. Vet Microbiol 180(1-2): 96-102.

12. Anikhovskaya IA, Kubatiev AA, Salakhov IM, Tebloev KI, Yakovlev MY (2015) Changes of endotoxin concentration in blood serum in patients with uncomplicated acute myocardial Q-infarction. Patol Fiziol Eksp Ter 3: 55-61.

13. Romanovsky AA, Ivanov AI, Lenczowski MJP, Kulchitsky VA, Van-Dam A-M, et al. (2000) Lipopolysacharide transport from the peritoneal cavity to the blood: is it controlled by the vagus nerve? Autonom Neurosci 85(1-3): 133-140.

14. Dogan MD, Kulchitsky VA, Patel S, Petervari E, Szekely M, et al. (2003) Bilateral splanchnicotomy does not affect lipopolysaccharide-induced fever in rats. Brain Res 993(1-2): 227-229.

15. Liu E, Lewis K, Al-Saffar H, Krall CM, Singh A, et al. (2012) Naturally occurring hypothermia is more advantageous than fever in severe forms of lipopolysaccharide and E coli induced systemic inflammation. Am J Physiol Regul Integr Comp Physiol 302(12): 1372-1383. 


\section{ISSN: 2574-1241}

DOI: $10.26717 / B J S T R .2018 .08 .001630$

Vladimir Kulchitsky. Biomed J Sci \& Tech Res

(c) (P) This work is licensed under Creative

Submission Link: https://biomedres.us/submit-manuscript.php

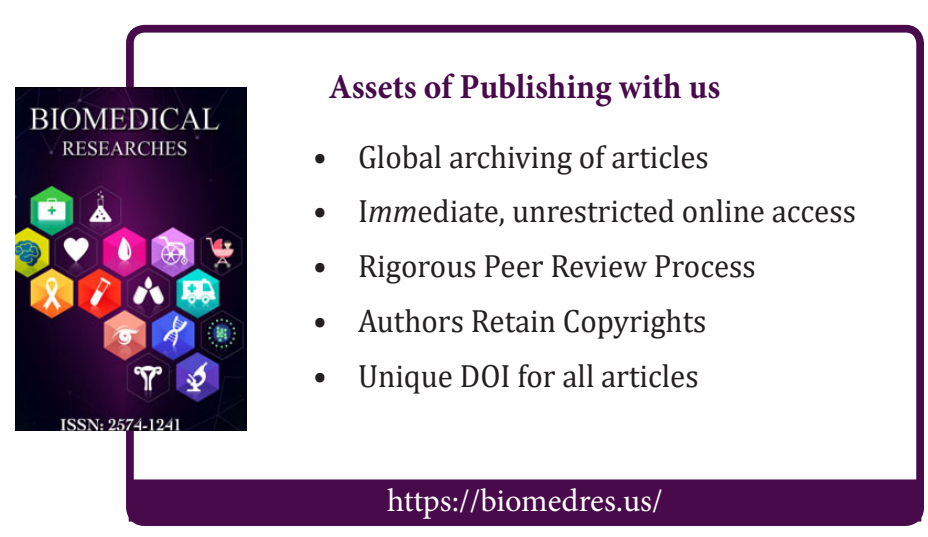

